\title{
Dedicated Trigger for Highly lonising Particles at ATLAS
}

\author{
Akshay Katre* (On behalf of the ATLAS Collaboration) \\ Particle Physics department, University of Geneva, 1211 Geneva 4, Switzerland \\ E-mail: akshay.kecern.ch
}

In 2012, a novel strategy was designed to detect signatures of Highly Ionising Particles (HIPs) such as magnetic monopoles, dyons or $Q$-balls with ATLAS. A dedicated trigger was developed and deployed for proton-proton collisions at a centre of mass energy of $8 \mathrm{TeV}$. A distinct algorithm is developed using the Transition Radiation Tracker (TRT) system. The high threshold (HT) readout capability of the TRT is used to distinguish HIPs from other background processes. The trigger requires significantly lower energy depositions in the electromagnetic calorimeters and is thereby capable of probing a larger range of HIP masses and charges.

A description of the algorithm for this newly developed trigger is presented, along with a comparative study of its performance during the 2012 data-taking period with respect to previous efforts.

The European Physical Society Conference on High Energy Physics 22-29 July 2015

Vienna, Austria

${ }^{*}$ Speaker. 


\section{Introduction}

The CERN Large Hadron Collider (LHC) is capable of accessing the multi-TeV energy range that enables the exploration of previously uncharted territories of particle physics. New stable massive particles would represent dramatic deviations from current Standard Model predictions. Searches for long-lived particles with highly ionising (HIPs) signatures have been carried out at collider experiments such as OPAL [1], CDF [2] and ATLAS [3, 4]. HIP signatures can be attributed to particles in theories beyond the Standard Model, such as magnetic monopoles, dyons, strange quark matter, $Q$-balls, and stable microscopic black-hole remnants [5].

Previous searches for HIPs at ATLAS relied on standard electromagnetic (EM) calorimeter triggers whose transverse energy $\left(E_{\mathrm{T}}=E \sin \theta^{1}\right)$ thresholds were gradually increased ${ }^{2}$, thus hampering HIP search efficiencies. Furthermore, the standard EM triggers implicitly required energy deposition in the second layer of the EM calorimeter, thereby unable to capture HIPs that stop in the presampler or the first layer (EM1) of the EM Calorimeter.

\section{Signatures of highly ionising particles}

The signatures of HIPs differ from those of known objects (e.g. electrons, muons and jets) reconstructed at ATLAS and would be missed by analyses that rely on such objects. The HIPs are characterised by an ionisation energy loss which is orders of magnitude greater than minimum ionising particles while propagating through matter [6].
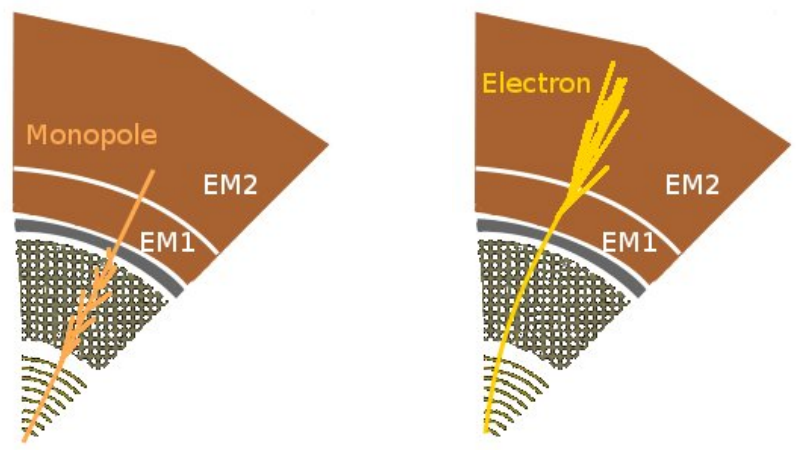

Figure 1: A schematic representation a monopole (left) and an electron (right) losing their energy when traversing through and stopping in the ATLAS detector (shown only up to the EM calorimeter)

At ATLAS, searches for HIPs are performed by considering a particle producing a region of high ionisation density in the transition radiation tracker (TRT) and slowing down and stopping in the EM calorimeter. Energy loss by bremsstrahlung and $e^{+} e^{-}$pair production are negligible for

\footnotetext{
${ }^{1}$ ATLAS uses a right-handed coordinate system with its origin at the nominal interaction point (IP) in the centre of the detector and the $z$-axis along the beam pipe. The $x$-axis points from the IP to the centre of the LHC ring, and the $y$-axis points upward. Cylindrical coordinates $(r, \phi)$ are used in the transverse plane, $\phi$ being the azimuthal angle around the beam pipe. The pseudorapidity is defined in terms of the polar angle $\theta$ as $\eta=-\ln \tan (\theta / 2)$.

${ }^{2}$ In order to control the rate while the LHC delivered luminosity was growing
} 
HIPs and thus the ionisation energy deposit in the EM calorimeter is narrower than that associated with electrons and photons, which induce an EM shower. An illustrative diagram representing this phenomenon is shown in Fig 1. The signature of the HIPs in the TRT is exploited by the dedicated HIP trigger to suppress backgrounds and achieve high efficiency for HIP signals.

\section{Highly ionising particle trigger}

The Run-1 ATLAS trigger system consists of a three level system, the first of which is a hardware based system, Level-1 (L1) that uses the energy information provided by the EM and hadronic calorimeters. Trigger towers of granularity $0.1 \times 0.1$ in $\Delta \phi \times \Delta \eta$ are built in these calorimeters around the cells with maximum energy deposits, as shown in Fig. 2. The L1, in the case of the HIP trigger, selects candidates based on the transverse energy deposited in the EM calorimeter $\left(E_{\mathrm{T}}^{\mathrm{EM}}>18 \mathrm{GeV}\right)$ and rejects candidates if more than $1 \mathrm{GeV}$ of energy is deposited in the hadronic calorimeter. The hardware based trigger is ideally developed for triggering on candidates that stop in the EM calorimeter.

The position of the $\mathrm{L} 1$ deposits defines the Region of Interest (RoI) which is then used by the latter stages of the trigger system. The Level-2 (L2) and Event-Filter (EF) stages of the trigger system are software based, where the algorithms accesses data from a limited geometrical region centred around the L1 RoI candidate position.

At L2, simplified tracking and reconstruction algorithms are possible and the HIP trigger uses the TRT hit information associated with the L1 RoI. A wedge with 20 bins of 0.01 radians in $\phi$ centred around the $\phi$ position of the RoI is built. The number of high threshold (HT) TRT hits is computed in each of these bins. Three bins (corresponding to a wedge of size 0.030 radians in $\phi$ ) centred around the bin with the maximum number

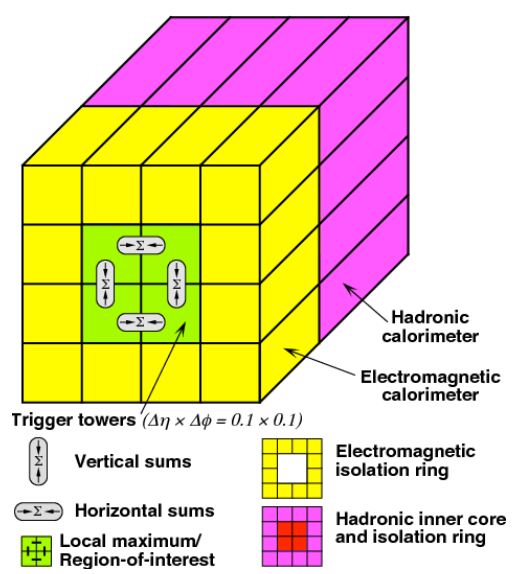

Figure 2: Illustration of the standard L1 trigger towers of granularity $0.1 \times 0.1$ in $\Delta \phi \times \Delta \eta$ around the cells with maximum energy deposits. Taken from Ref. [7] of HT TRT hits are chosen to build two sensitive variables, namely, the number $\left(N_{\mathrm{HT}}^{\text {Trigger }}\right)$ and fraction $\left(f_{\mathrm{HT}}^{\text {Trigger }}\right)$ of HT TRT hits. The two sensitive variables are powerful discriminants against Standard Model background processes, as shown in Fig. 3 for the $f_{\mathrm{HT}}^{\text {Trigger }}$ variable. The high ionisation from HIPs tend to produce higher $f_{\mathrm{HT}}^{\text {Trigger }}$ values compared to the Standard Model background processes.

\section{Performance}

The HIP trigger was deployed at ATLAS where it collected data from September 2012 till the end of Run 1 of the LHC. The rate of the trigger was maintained at less than $1 \mathrm{~Hz}$ during this period. Fig. 4 shows the rate of the HIP trigger in a typical run as a function of the instantaneous 


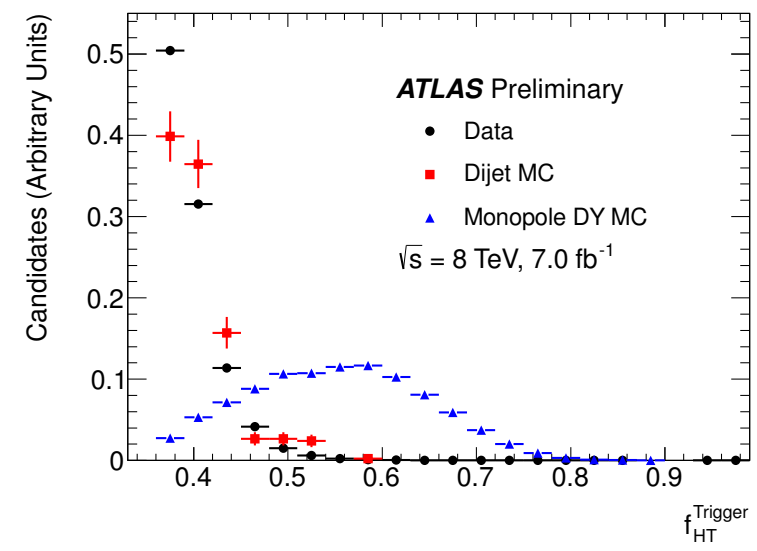

Figure 3: The fraction of high threshold TRT hits computed in a wedge in $\phi$ of size 0.03 centred around the bin with the highest number of HT hits in the TRT. A cut is placed on this variable at 0.37 to optimise for high signal efficiency and low rate. In data, the HIP trigger fires on dijet events that rarely produce large number of HT hits in the TRT but have large cross-sections. A typical Drell-Yan monopole signal sample [6] produces high fraction values. The histograms are normalised to an integral of 1. Taken from Ref. [8].

luminosity. The instantaneous luminosity of the run decreases as the run progresses and we see a gradual increase in the rate of the trigger as the run progresses.

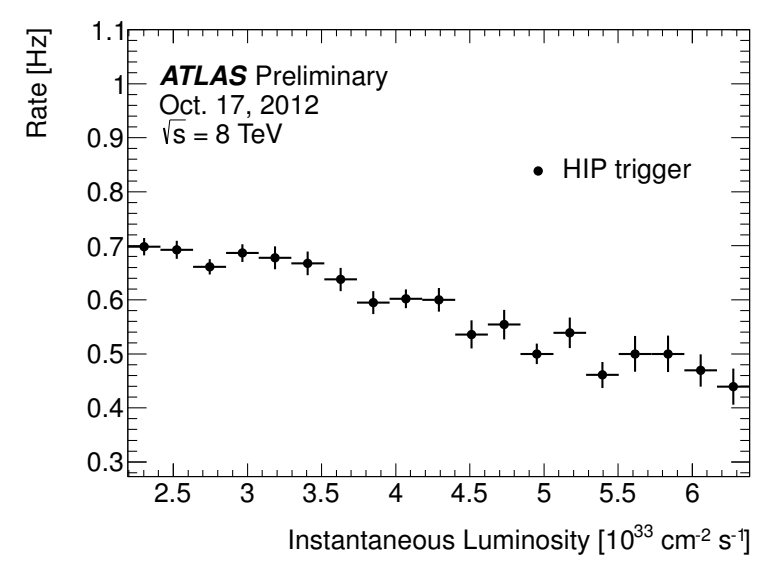

Figure 4: The rate of the HIP trigger during a typical run in 2012. The plot shows the rate of the HIP trigger as a function of instantaneous luminosity of the run. The overall rate of the trigger during all runs was less than a $\mathrm{Hz}$ and is the highest at the end of the run (low values of instantaneous luminosity) and lower at the beginning of the run (higher values of instantaneous luminosity). Taken from Ref. [8]

The number of interactions per bunch crossing (pile-up, $\langle\mu\rangle$ ) decreases as the instantaneous luminosity of the run decreases. The rate of the HIP trigger is dependent on the pile-up. At high pile-up there are more low $\mathrm{p}_{\mathrm{T}}$ objects that produce a large number of low threshold hits that dilute the $f_{\mathrm{HT}}^{\text {Trigger }}$ of the trigger, thereby increasing the probability of the event being rejected.

Fig. 5 shows the number of TRT hits from the trigger as a function of pile-up for a simulated 


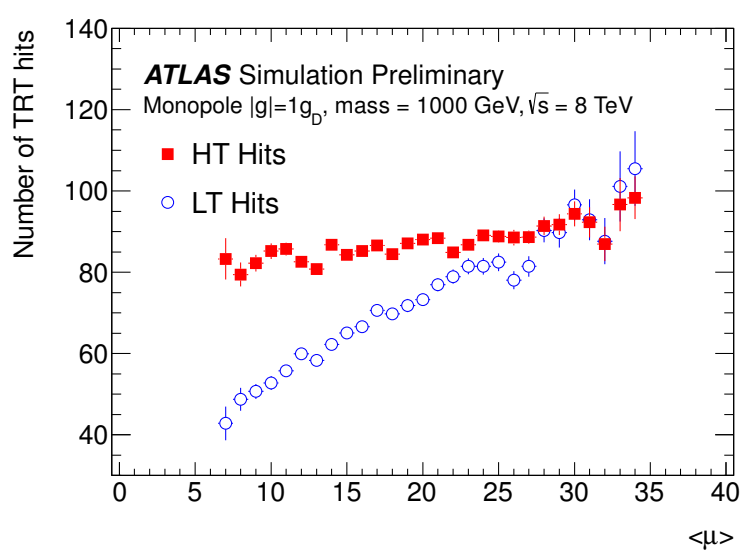

Figure 5: The number of TRT hits is shown in a typical Drell-Yan monopole signal sample [6] as a function of average pile-up $(\langle\mu\rangle)$. At higher $\langle\mu\rangle$ there are more low $\mathrm{pT}$ candidates in the event that produce a larger number of low threshold TRT hits. The number of HT hits originate primarily from the monopole traversing through the detector and it remains approximately constant. Taken from Ref. [8].

monopole samples overlaid with the pile-up conditions in a 2012 run. As expected from a signal sample, the number of HT TRT hits remains constant while the number of low threshold hits (largely from low $\mathrm{p}_{\mathrm{T}}$ objects) increases with pile-up. The trigger is self regulating, in the sense that if the luminosity of the runs is increased, the rate of the trigger will keep decreasing due to higher pile-up.

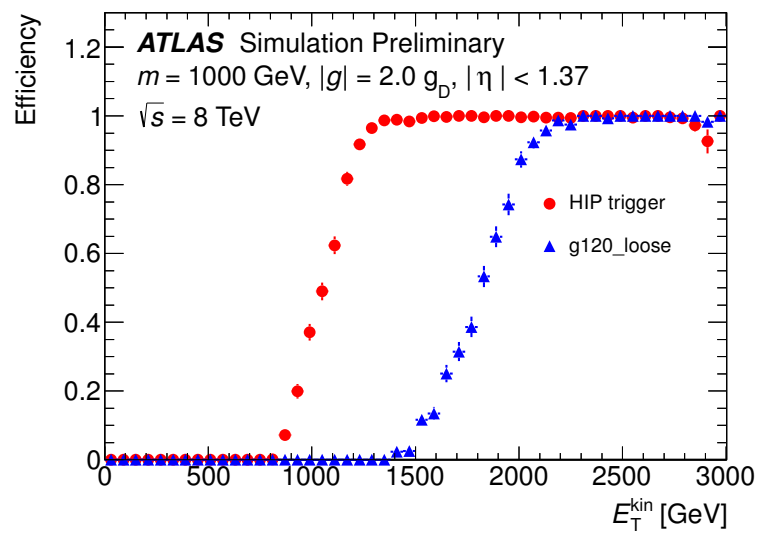

Figure 6: The trigger efficiency as a function of initial transverse kinetic energy for a single particle monopole of mass $1000 \mathrm{GeV}$, charge $2 \mathrm{gD}$ for the HIP trigger and a standard photon trigger in the barrel of the ATLAS detector. Taken from Ref. [8].

\section{Results}

The dedicated HIP trigger is capable of selecting candidates that stop in the first two layers of the EM calorimeter, unlike standard electron/ photon triggers. In addition, the transverse energy 
thresholds are much lower $(\sim 18 \mathrm{GeV})$ for the HIP trigger compared to previous analysis that used standard electron/ photon triggers $(\sim 120 \mathrm{GeV})$. These factors contribute to a greater acceptance for HIPs with the help of the dedicated trigger, as seen in Fig. 6 for a monopole carrying twice the Dirac charge $\left(g_{\mathrm{D}}\right)$. This is also the first time at ATLAS that a monopole of charge $2 g_{\mathrm{D}}$ is probed with a non-negligible acceptance.

In 2015, with higher centre of mass energies probed at the LHC, the transverse energy thresholds for standard triggers will be raised further, making a strong case for the dedicated HIP trigger. Narrower wedge sizes optimised for dealing with higher pile-up conditions will help maintain both a high signal efficiency and a low background rate.

\section{References}

[1] G. Abbiendi et al. [OPAL Collaboration], Phys. Lett. B 663 (2008) 37 [arXiv:0707.0404 [hep-ex]].

[2] A. Abulencia et al. [CDF Collaboration], Phys. Rev. Lett. 96 (2006) 201801 [hep-ex/0509015].

[3] ATLAS Collaboration, Phys. Lett. B 698 (2011) 353 [arXiv:1102.0459 [hep-ex]].

[4] ATLAS Collaboration, Phys. Rev. Lett. 109 (2012) 261803 [arXiv:1207.6411 [hep-ex]].

[5] B. Acharya et al. [MoEDAL Collaboration], Int. J. Mod. Phys. A 29 (2014) 1430050 [arXiv:1405.7662 [hep-ph]].

[6] A. De Roeck, A. Katre, P. Mermod, D. Milstead and T. Sloan, Eur. Phys. J. C 72 (2012) 1985 [arXiv:1112.2999 [hep-ph]].

[7] ATLAS Collaboration, 2008 JINST 3 S08003

[8] https://twiki.cern.ch/twiki/bin/view/AtlasPublic/EgammaTriggerPublicResults 